\title{
Commentaries
}

\section{Giving carditis back to the heart}

Anatomically vague (it was named after its proximity to the heart), histologically undistinguished (its mucosa is usually described as being "similar to the mucosa of the antrum") and functionally considered a drab territory that connects two well characterised segments of the digestive system, the gastric cardia has long been ignored by gastroenterologists, pathologists, and physiologists alike.

Suddenly, in the past few years, this neglected Grenz zone has been catapulted to the centre of the gastroenterological stage. Another spin-off of Helicobacter pylor?? In a sense, yes. But, whereas the wily bacterium is blamed for a long list of calamities occurring in the remainder of the stomach, duodenum, and other systems, in the cardia it is portrayed as a protector of mucosal integrity. ${ }^{1}$ An implausible defensor mucosae.

During the past few decades a dramatic rise in the incidence of adenocarcinoma of the cardia has been reported in the very populations in which the incidence of gastric cancer has been decreasing. ${ }^{2}$ The latter trend has been explained as a consequence of the declining prevalence of $H$ pylori infection. However, the increased incidence of cancer of the cardia is paradoxical: why should this minuscule ill-defined portion of the proximal compartment of the stomach behave differently from its more distal areas? One possible explanation is that the lack of a consensus on what the cardia area is, the difficulty of establishing the precise site of origin of tumours of the gastro-oesophageal junction, and various inaccuracies in tumour coding in cancer registries may have unwittingly conspired to inflate trends in cardia cancer. ${ }^{3}$ In fact, most cancers classified as cardia tumours may arise in Barrett's epithelium in the distal oesophagus. The rise in acid reflux and Barrett's oesophagus in Western populations is probably related to dietary trends (more dietary fat), which have increased the prevalence of obesity, ${ }^{4}$ and by the increased gastric acid production made possible by the decreasing prevalence of $H$ pylori induced atrophic gastritis. ${ }^{15}$ Recently, more investigators are inclined to accept the oesophageal origin of junctional tumours and have called for a consolidation of cancer arising in this area into a single clinicopathological entity. ${ }^{67}$ Nevertheless, discovering what happens in the cardia may be important, or even crucial, to understanding the events taking place at the junction.

In 1994 we tackled the issue of cardiac involvement by $H$ pylori $^{8}$ : in subjects with $H$ pylori elsewhere in the stomach the mucosa of the cardia was almost always directly affected, and the inflammatory responses in the cardia and the antrum were similar in both quality and intensity. Our study did not suggest that the cardia was a compartment of the stomach affected by $H$ pylori in a distinctive fashion. Therefore, we did not see the need for a special term (carditis?) any more than we believed that inflammation of the pylorus should be called pyloritis or the inflammation of the greater curvature grand curvitis. In 1996 Riddell introduced the term carditis, ${ }^{9}$ hitherto used exclusively in reference to inflammatory diseases of the heart, to connote "inflammation of the gastric cardia." Since then, the perception of an epidemic of junctional cancer, the sugges- tions that $H$ pylori eradication may result in increased acid reflux, and perhaps the relative virginity of the territory have provided the momentum for numerous investigations of the prevalence, nature, and importance of inflammation in the gastric cardia.

The results of these studies are remarkable for their disparity. Goldblum and colleagues ${ }^{10}$ found inflammation of the cardia in 23 patients with gastro-oesophageal reflux disease (GORD); only one of these patients was not infected with $H$ pylori. Similarly, El-Serag et al found no instances of inflammation of the cardia not associated with $H$ pylori infection in 302 patients with reflux oesophagitis. ${ }^{11}$ In contrast, Chen et al found "some carditis" in $107(92 \%)$ of 116 unselected subjects. ${ }^{12}$ Forty per cent of these subjects with moderate and $57 \%$ of those with severe carditis had $H$ pylori infection. The authors focused on these data to conclude that carditis is significantly associated with $H$ pylori active gastritis, but not with symptoms or signs of gastro-oesophageal reflux. The same figures, however, show that $60 \%$ of individuals with moderate and $43 \%$ of those with severe inflammation in the cardia did not have $H$ pylori infection. In Voutilainen's Finnish series of 110 patients with junctional intestinal metaplasia, 101 (92\%) had cardia gastritis, but only 50 (46\%) had $H$ pylori infection; of the 1009 controls without junctional intestinal metaplasia $737(73 \%)$ had carditis, but only 363 (36\%) had H pylori infection. ${ }^{13}$ The study by Bowrey et al in this issue (see page 798) confirms that subjects with GORD have a high prevalence of cardia inflammation, even in the carefully documented absence of a $H$ pylori infection.

Thus, we have several major discrepancies to explain. Why do some authors detect essentially no inflammation in the cardia of $H$ pylor $i$-free subjects, ${ }^{10}{ }^{11}$ whereas others find that a large portion of uninfected subjects have isolated gastritis of the cardia (Bowrey et al) $?^{12}{ }^{13} \mathrm{~A}$ second issue raised by these data is the relation between cardia inflammation and reflux: some authors believe that inflammation in the cardia and evidence of GORD (Bowrey et al) ${ }^{13}$ go hand in hand, whereas other investigators fail to see evidence for such a correlation. ${ }^{10}{ }^{11}$ Finally, if we do accept the existence of an entity to be provisionally called "non- $H$ pylori isolated carditis," we may have to appose the defeating qualifier "idiopathic" to indicate our ignorance about its aetiology. Having now created the novel entity "idiopathic non-H pylori isolated carditis" we must ask ourselves what its importance might be. The practical corollary of this theoretical question is, of course, whether it is sensible for endoscopists to go to the extra effort and retroflex the endoscope to obtain one or two biopsy specimens from the cardia. In the present state of knowledge, the histopathological answers they receive will be very unlikely to influence patient management.

Geographical differences are unlikely to explain the disparities found in the studies outlined earlier. Although the ethnic background of the subjects is rarely stated or taken into account in the analyses, most patients in Voutilainen's series were certainly Finns, ${ }^{13}$ the majority of Bowrey's patients were likely to be Welsh, and Chen's subjects were Americans with various percentages of the ethnic groups that constitute the population of Boston. ${ }^{12}$ Yet, these diverse groups had comparable prevalence of "idiopathic 
non-H pylori isolated carditis". Criteria used to define carditis are also unlikely to explain the substantial differences found in different populations. Most investigators have made a reference to their pathologists' use of the updated Sydney System. ${ }^{14}$ Even taking into account interobserver variation among pathologists, the discrepancies seem too wide to be explained by this interpretative variable alone. The inevitable conclusion is that, at this point, we do not have the necessary information to even guess the true prevalence of non- $H$ pylori carditis in different populations, we do not know what it means, and have no idea what should be done about it. In this situation, the only sensible course of action is to encourage the performance of well designed studies, of which Bowrey's article is an example, in geographically and ethnically diverse populations of clinically well defined subjects. Data on the intensity and topographical distribution of gastritis ought to be available for each subject, so that correlations with inflammation in the cardia can be made. The combined information derived from such studies may eventually help to remove "idiopathic" from the provisional name given to the non- $H$ pylori carditis. It might even suggest that the term carditis should be given back to the heart.

Department of Pathology 113

VA Medical Centre and Baylor College of Medicine, 2002 Holcombe Blvd,

Houston, TX 77030, USA
1 Blaser MJ. Hypothesis: the changing relationships of Helicobacter pylori and humans: implications for health and disease. F Infect Dis 1999;179:152330.

2 Blot WJ, Devesa SS, Kneller RW, et al. Rising incidence of adenocarcinoma of the esophagus and gastric cardia. $\mathcal{F} A M A$ 1991;265:1287-9.

3 Ekström AM, Signorello LB, Hansson LE, et al. Evaluating gastric cancer misclassification: a potential explanation for the rise in cardia cancer incidence. $\mathcal{F}$ Natl Cancer Inst 1999;91:786-90.

4 Lagergren J, Bergström R, Nyren O. Association between body mass and adenocarcinoma of the esophagus and gastric cardia. Ann Intern Med 1999; 130:883-90.

5 Labenz J, Tillenburg B, Peitz U, et al. Effect of curing Helicobacter pylori infection on intragastric acidity during treatment with ranitidine in patients with duodenal ulcer. Gut 1997;41:33-6.

6 Dolan K, Sutton R, Walker SJ, et al. New classification of oesophageal and gastric carcinomas derived from changing patterns in epidemiology. $\mathrm{Br} \mathcal{F}$ Cancer 1999;80:834-42.

7 Wijnhoven BP, Siersema PD, Hop WC, et al. Adenocarcinomas of the distal oesophagus and gastric cardia are one clinical entity. Rotterdam Oesophageal Tumour Study Group. Br F Surg 1999;86:529-35.

8 Genta RM, Huberman RM, Graham DY. The gastric cardia in Helicobacter pylori infection. Hum Pathol 1994;25:915-19.

9 Riddell RH. The biopsy diagnosis of gastroesophageal reflux disease, "carditis", and Barrett's esophagus, and sequelae of therapy. Am F Surg Pathol 1996;20(suppl 1):S31-50.

10 Goldblum JR, Vicari JJ, Falk GW, et al. Inflammation and intestinal metaplasia of the gastric cardia: the role of gastroesophageal reflux and $\mathrm{H}$. pylori infection. Gastroenterology. 1998;114:633-9.

11 El-Serag HB, Sonnenberg A, Jamal MM, et al. Characteristics of intestinal metaplasia in the gastric cardia. Am f Gastroenterol 1999;94:622-7.

12 Chen YY, Antonioli DA, Spechler SJ, et al. Gastroesophageal reflux disease versus Helicobacter pylori infection as the cause of gastric carditis. Mod Pathol 1998;11:950-6.

13 Voutilainen M, Farkkila M, Juhola M, et al. Specialized columnar epithelium of the esophagogastric junction: prevalence and associations. The Central Finland Endoscopy Study Group. Am f Gastroenterol 1999;94:913-18.

14 Dixon MF, Genta RM, Yardley JH, et al. Classification and grading of gastritis. The updated Sydney system. International workshop on the histopathology of gastritis, Houston 1994. Am F Surg Pathol 1996;20: $1161-81$.

\section{Colon cancer: the shape of things to come}

Konishi et al (see page 818) have documented morphological findings in 4147 colorectal neoplasms detected colonoscopically in a consecutive series of 5025 average risk subjects. The importance of their report lies in the understated and sobering perspective it brings to the concept of de novo colorectal cancer. They used high resolution magnifying endoscopy to detect, remove, and classify all epithelial neoplasms. The neoplasms were grouped as mucosal lesions (3605), submucosal cancers (91), and advanced cancers (451), although the advanced cancers were not considered further. The mucosal lesions included 3353 adenomas and 252 intramucosal carcinomas. In the West, the latter would be classified as adenomas with high grade dysplasia $^{1}$ and for the purposes of this study were grouped with the adenomas, and are subsequently referred to as such.

The neoplasms were also grouped into depressed and non-depressed, the latter including flat, slightly raised, and polypoid lesions. The rationale for this was the previous demonstration of de novo cancer associated genetic changes specifically within early depressed cancer. ${ }^{2}$ The findings seem to ratify this decision. Among the 86 non-depressed submucosal cancers, residual adenoma was found in $63(73 \%)$; it is conceivable that an adenomatous component was destroyed in the remainder. None of the depressed submucosal cancers (five out of the 91 found) showed residual adenoma. If it is assumed that there was no precursor lesion in these five cases, it must nevertheless be accepted that de novo carcinoma is uncommon, even in
Japan. The distribution of these putative de novo submucosal cancers is also of interest: three in the right colon ( $17 \%$ of right sided submucosal cancers), two in the left colon ( $5 \%$ of left sided submucosal cancers), and none in the rectum.

The fact that depressed submucosal cancers are more common in the right colon and show a low frequency of K-ras mutation ${ }^{3}{ }^{4}$ fits with the recent description of DNA microsatellite instability in flat appearing cancers of the proximal colon. ${ }^{5} \mathrm{~K}$-ras mutation is uncommon in cancers showing extensive microsatellite instability (MSI-H phenotype). ${ }^{6}$ As adenomas in hereditary non-polyposis colorectal cancer (HNPCC), which are often MSI-H, are thought to show an accelerated progression to cancer, ${ }^{7}$ it is possible that the lack of residual adenoma in flat or depressed carcinoma is explained by malignant conversion of an adenoma when it is still small and therefore susceptible to complete obliteration.

The study by Konishi et al offers indirect evidence that depressed carcinoma may indeed develop within depressed adenoma. The percentage of lesions with a depressed appearance is similar for both adenoma (4\%) and carcinoma (5\%). Furthermore, depressed neoplasms (benign and malignant) are vanishingly rare in the rectum and most common in the proximal colon. To these points may be added the earlier observations of infrequent K-ras mutation in flat or depressed adenoma as well as carcinoma. ${ }^{3} 8$

It has been known for many years that colorectal adenoma and cancer are distributed differently in the colon. Autopsy studies show a relatively even distribution of adenomas throughout the colon whereas cancer is more frequent in the distal colon and rectum. ${ }^{9}$ Presumably, the microenvironment in the distal colon and rectum favours multistep neoplastic progression (or promotion). Konishi and colleagues support this notion by showing a higher 
frequency of $>10 \mathrm{~mm}$ polyps, a higher frequency of villosity among the non-depressed adenomas of the distal colon and rectum, and a higher carcinoma:adenoma ratio in the distal colon and rectum.

The often repeated assertion that proximal and distal large bowel cancers are fundamentally different is alluded to in this study. It seems that the main basis for this observation is the predilection of MSI-H cancers for the proximal colon. ${ }^{10}$ As noted previously, this fact may explain the distribution of flat or depressed neoplasms. Nevertheless, other factors may determine the growth characteristics of colorectal neoplasms. Mechanical shearing forces may be maximal in the distal colon and rectum, encouraging mucosal prolapse and the development of a polypoid mass. Less mundane explanations may lie in the growth characteristics of neoplastic crypts. Crypt elongation and branching will produce an expansile lesion that is more likely to become exophytic. When neoplastic crypts remain short, branch infrequently, and spread laterally, lesions will be flat or depressed. At this time, however, there is limited information on the mechanisms underlying the growth of neoplasms.

This study supports the adenoma-carcinoma concept, highlighting this as the major morphogenetic pathway for colorectal cancer in the left colon and rectum. The de novo pathway, by contrast, is uncommon and operates mainly in the proximal colon. Even this suggestion may be overstating the importance of the de novo pathway if the explana- tion for an apparent de novo origin lies in the rapid conversion of flat or depressed adenomas showing DNA microsatellite instability.

Department of Pathology,

J R JASS

The University of Queensland Medical School,

Herston, Queensland 4006, Australia

1 Schlemper RJ, Itabashi M, Kato Y, et al. Differences in the diagnostic criteria used by Japanese and Western pathologists to diagnose colorectal carcinoma. Cancer 1998;82:60-9.

2 Kato S, Fujii T, Oda Y, et al. Difference of genetic alterations between polypoid, flat and depressed growth-type colorectal cancers [abstract]. Gastroenterology 1997;112:A589.

3 Fujimori T, Satonaka K, Yamamura-Idei Y, et al. Non-involvement of ras mutations in flat colorectal adenomas and carcinomas. Int $\mathcal{f}$ Cancer 1994;57:51-5.

4 Minamoto T, Sawagushi K, Mai M, et al. Infrequent K-ras activation in superficial-type (flat) colorectal adenomas and carcinomas. Cancer Res 1994;54:2841-4

5 Okamoto T, Konishi F, Kojima M, et al. Significance of microsatellite instability in different types of early stage non-familial cancers. Dis Colon Rectum 1998;41:1385-91.

6 Konishi M, Kikuchi-Yanoshita R, Tanaka K, et al. Molecular nature of colon tumors in hereditary non-polyposis colorectal cancer, familial polyposis, tumors in hereditary non-polyposis colorectal cancer, familial

7 Jass JR, Stewart SM. Evolution of hereditary non-polyposis colorectal cancer. Gut 1992;33:783-6.

8 Yamagata S, Muto T, Uchida Y, et al. Lower incidence of K-ras codon 12 mutation in flat colorectal adenomas than in polypoid adenomas. $f p n f$ Cancer Res 1994;85:147-51.

9 Jass JR, Young PJ, Robinson EM. Predictors of presence, multiplicity, size and dysplasia of colorectal adenomas. A necropsy study in New Zealand. Gut 1992;33:1508-14.

10 Jass JR, Do K-A, Simms LA, et al. Morphology of sporadic colorectal cancer with DNA replication errors. Gut 1998;42:673-9.

\section{Cryptic messages in FAP}

A series of remarkable clinical, epidemiological, and laboratory studies has shown that aspirin and other nonsteroidal anti-inflammatory drugs (NSAIDs) such as sulindac, can prevent the development of colorectal cancer and cause regression of pre-existing adenomas. ${ }^{1}$ Aspirin is one of the most potent chemopreventive agents against colorectal cancer. However, its mechanism for causing a reduction in colorectal cancer risk and mortality is unknown. ${ }^{2}$ The anti-inflammatory properties of NSAIDs are thought to reside in their ability to inhibit prostaglandin production at sites of inflammation.

Cyclooxygenase (COX) is a key enzyme in the production of prostaglandins, and aspirin can inhibit its activity directly. Initially, COX was thought to be a single enzyme, but in 1991 a second COX isoform was discovered (COX-2) which is induced by cytokines, growth factors, and tumour promoters. ${ }^{3}$ COX-1 is expressed constitutively in many tissues and is inhibited by aspirin and other commonly used NSAIDs. Contrastingly, COX-2 can be induced and expressed at high levels in most colorectal cancers. ${ }^{4-6}$ Both isoforms catalyse the conversion of arachidonic acid to a variety of eicosanoid products, including prostaglandins. Inhibition of COX-1 by NSAIDs is a major cause of NSAID associated gastropathy. ${ }^{7}$ However, COX-2 is undetectable or barely expressed in the normal stomach and its inhibition does not cause peptic ulceration. ${ }^{89}$ Recently, a number of highly specific COX-2 inhibitors has been developed which promise to prevent colorectal cancer without incurring serious side effects in the rest of the gastrointestinal tract. ${ }^{1011}$

COX-2 plays a major role in the development of adenomas. Min and $\mathrm{APC}^{4716}$ mice, which have mutations in the
APC gene analogous to those in patients with familial adenomatous polyposis (FAP), have significantly fewer adenomas if COX-2 is inhibited pharmacologically or removed by genetic manipulation. ${ }^{12}$ Furthermore, COX-2 contributes to the malignant phenotype as it promotes tissue invasion, angiogenesis, and may even create areas of immune privilege within the tumour. ${ }^{13-15}$

How does aspirin prevent the development of adenomas and cause the regression of pre-existing adenomas? In a landmark randomised controlled trial, Giardiello and colleagues have already shown that sulindac can cause the regression of pre-existing colonic adenomas in patients with familial adenomatous polyposis. ${ }^{16}$ In their current paper (see page 822) the authors confirmed that sulindac decreases polyp numbers in patients with FAP. They then evaluated apoptosis along the long axis of the crypt in biopsy samples of normal rectal mucosa from patients treated with sulindac for three months, finding that sulindac increases apoptosis at the surface of the crypt, but decreases apoptosis at the base. These changes in the distribution of apoptosis cannot be explained by changes in the expression of genes known to regulate apoptosis-for example, Bcl-2, Bax, p53, or the cell cycle inhibitor p21. Colonic epithelial cells arise from stem cells located at the base of the crypt which then migrate up to the intercrypt table at the surface, where they are shed. There is a constant low rate of apoptosis at the base of the crypt which may regulate the number of cells migrating towards the surface. Apoptosis may also contribute to the shedding process at the top of the crypt. However, it is unclear whether apoptosis is the regulated event which causes cell shedding or whether it is merely secondary to cleavage of cellular integrins from their ligands in the extracellular matrix. The significance of the change in distribution of apoptosis from the base of the crypt towards the surface during treatment with sulindac is unclear and further 
studies will be needed. However, Keller et al suggest that this change in ratio of apoptosis between the crypt base and surface may be a useful intermediate biomarker for studying the efficacy of chemopreventive agents of colorectal tumorigenesis. This is a bold claim as the accurate assessment of apoptosis in human colonic biopsy samples is notoriously hazardous. The authors report that the mean number of crypts counted in each patient was 12.74 . Apoptosis is so heterogeneous that to obtain reproducible data in the mouse colon, at least 200 perfectly orientated half crypt sections must be quantified. Nevertheless, the concept of NSAIDs increasing cell shedding at the mucosal surface is an appealing one and should stimulate future studies aimed at validating its usefulness as a biomarker in humans.

Department of Medicine,

University of Liverpool,

Liverpool, UK

A WATSON

Department of Medicine, GI; MCN C-2104,

Vanderbilt University Medical Centre,

Nashville, TN 37232-1080, USA

1 Smalley W, DuBois RN. Colorectal cancer and nonsteroidal antiinflammatory drugs. Adv Pharmacol 1997;39:1-20.

2 Williams CS, Smalley W, DuBois RN. Aspirin use and potential mechanisms for colorectal cancer prevention. $\mathcal{F}$ Clin Invest 1997;100:1-5.

3 Williams CW, DuBois RN. Prostaglandin endoperoxidase synthase: why two isoforms? Am f Physiol 1996;270:G393-400.
4 Eberhart CE, Coffey RJ, Radhika A, et al. Up-regulation of cyclooxygenase-2 gene expression in human colorectal adenomas and adenocarcinomas. Gastroenterology 1994;107:1183-8.

5 Sano H, Kawahito Y, Wilder RL, et al. Expression of cyclooxygenase-1 and -2 in human colon cancer. Cancer Res 1995;55:3785-9.

6 Kargman S, O'Neill G, Vickers P, et al. Expression of prostaglandin G/H synthase-1 and -2 protein in human colon cancer. Cancer Res 1995;55: 2556-9.

7 Scheiman JM. NSAIDs, gastrointestinal injury and cytoprotection. Gastroenterol Clin North Am 1996;25:279-98.

8 Goldstein JL, Agrawal NM, Silverstein F, et al. Celecoxib is associated with a significantly lower incidence of clinically significant upper gastrointestinal (UGI) events in osteoarthritis and rheumatoid arthritis patients compared to NSAIDs [abstract]. Gastroenterology 1999;116:G0758.

9 Laine L, Hawkey C, Harper S, et al. Effect of the COX-2 specific inhibitor (C-2SI) Rofecoxib on ulcer formation: a double-blind comparison with ibuprofen and placebo [abstract]. Gastroenterology 1999;116:G0996.

10 Kawamori T, Rao CV, Seibert K, et al. Chemopreventive activity of celecoxib, a specific cyclooxygenase- 2 inhibitor, against colon carcinogenesis. Cancer Res 1998;58:409-12.

11 Reddy BS, Rao CV, Seibert K. Evaluation of cyclooxygenase-2 inhibitor for potential chemopreventive properties in colon carcinogenesis. Cancer Res 1996;56:4566-9.

12 Oshima M, Dinchuk JE, Kargman SL, et al. Suppression of intestinal polyposis in $\mathrm{APC}^{\Delta 716}$ knockout mice by inhibition of prostaglandin endoperoxidase synthase-2 (COX-2). Cell 1996;87:803-9.

13 Tsujii M, DuBois RN. Alterations in cellular adhesion and apoptosis in epithelial cells overexpressing prostaglandin endoperoxidase synthase-2. Cell 1995;83:493-501.

14 Tsujii $M$, Kawano S, Tsujii S, et al. Cyclooxygenase regulates angiogenesis induced by colon cancer cells. Cell 1998;93:705-16.

15 Tsujii M, Kawano S, DuBois RN. Cyclooxgenase-2 expression in human colon cancer cells increases metastatic potential. Proc Natl Acad Sci USA 1997;94:3336-40.

16 Giardiello FM, Hamilton SR, Krush AJ, et al. Treatment of colonic and rectal adenomas with sulindac in familial adenomatous polyposis. $N$ Engl $\mathcal{F}$ Med 1993;328:1313-16.

\section{Post-cholecystectomy diarrhoea: a running commentary}

Since laparoscopic cholecystectomy eclipsed the open procedure in the early 1990 s there has been a worldwide increase in annual cholecystectomy rates of between $20 \%$ and $50 \% .^{12}$ This has occurred despite a lack of evidence that gallstone incidence has increased to the same degree. Laparoscopic cholecystectomy has a lower mortality rate than open cholecystectomy, but in view of the increased cholecystectomy rate, there may be no decrease in the total number of deaths associated with this operation. ${ }^{1}$ Changed indications for a safer procedure and patient or physician induced demand may explain the increased cholecystectomy rate. Patients and doctors may have a lower threshold for tolerance of gallstone associated symptoms than in the pre-laparoscopic era. As gallstones may be associated with a spectrum of symptoms, from none at all to those associated with life-threatening complications, in many instances the decision to operate may be arbitrary. This is especially so as the large majority of gallstones are asymptomatic, and these are best left undisturbed. ${ }^{3}$

Patient surveys two to 24 months after both open and laparoscopic cholecystectomy indicate that $40-50 \%$ of patients have persistence of one or two symptoms such as flatulent dyspepsia or dull abdominal pain, although $80-90 \%$ regard the operation as highly successful. ${ }^{4}$ Careful surveys carried out before and after surgery indicate that $13 \%$ of patients have persisting biliary type pain. ${ }^{6}$ Although it is easy to formulate a definition of biliary type pain for research purposes, the characteristics of pain caused by gallstones are still uncertain.?

Apart from the issues of procedure related mortality, changing indications for surgery, and symptom relief, there is much concern about post-laparoscopic cholecystectomy morbidity in the form of bile duct injuries. Major $(2.1 \% v$ $3.2 \%)$ and minor (5.9 $v 9.8 \%)$ postoperative complications occur less frequently with laparoscopic surgery. However, major bile duct injuries occur in about $0.33-0.5 \%$ of laparoscopic operations, compared with about $0.06 \%$ of open procedures. ${ }^{8}$

A small but important number of patients complain of diarrhoea in the long term after cholecystectomy. In a retrospective telephone survey of 148 patients who had had a cholecystectomy four years previously, 12\% described a major change in their bowel habit with more than three and up to six watery bowel movements daily. ${ }^{9}$ Other reports indicate that when patients are questioned about their bowel habits after cholecystectomy, between $5 \%$ and $8 \%$ answer that they have diarrhoea. Retrospective surveys, however, have drawbacks and questions remain regarding whether the cholecystectomy itself caused the diarrhoea, whether there was diarrhoea preoperatively, and whether what the patient describes as diarrhoea is real diarrhoea or just increased frequency of defecation. In this issue (see page 889) Hearing et al tackle these questions in a prospective survey of symptoms and measurements of bowel function before and after surgery in 106 adults. Patients estimated that their bowel frequency increased by a median of one bowel movement per week but measurements of interdefecatory interval and whole gut transit time using the Bristol Stool Form score did not change significantly. Two of three patients who had stated that they had diarrhoea usually or always before surgery reported a deterioration in their diarrhoea after surgery. In six patients who stated that they had diarrhoea usually or always postoperatively, five had perceived that they had less frequent diarrhoea or no diarrhoea at all preoperatively. Thus five patients either experienced a deterioration in their perception of diarrhoea or perceived that they had developed diarrhoea for the first time postoperatively. The situation is even more difficult to interpret as at least two of the five patients who had a perceived deterioration their 
diarrhoea or who had perceived that they had developed diarrhoea for the first time postoperatively, may have had irritable bowel syndrome pre- and postoperatively. Objective measurement of diarrhoea using interdefecatory interval and stool form in these five patients failed to show evidence of diarrhoea, except perhaps in one. Although Hearing et al did not show a difference in whole gut transit time after cholecystectomy using the stool form technique, it has been shown previously using a radio-opaque pellet method that whole gut transit time is shortened by about $20 \%$ as a consequence of the operation. ${ }^{9}$ Fortunately, cholecystectomy has a desired side effect for patients with perceived constipation preoperatively, most of whom feel relieved of this annoyance postoperatively.

What are the take home messages? $13-40 \%$ of patients have persisting abdominal pain after cholecystectomy although the vast majority regard their operation as a success. Up to $12 \%$ of post-cholecystectomy patients when questioned feel that they have diarrhoea as a consequence of their operation, and at least $4-5 \%$ of patients have a definite deterioration in their perceived diarrhoea or perceive that they have developed diarrhoea for the first time. Objective assessments postoperatively, however, rarely demonstrate new onset diarrhoea. Some of these patients may have the irritable bowel syndrome. Whether mean orocaecal transit time becomes faster postoperatively is still in doubt and this requires further study. Severe, high volume diarrhoea does not seem to occur as a consequence of cholecystectomy and if it does it is extremely rare. When faced with a patient with possible post-cholecystectomy diarrhoea, clinicians would be well advised to consider the irritable bowel syndrome and assess the situation objectively at the outset with records of defecation frequency, stool form, and assessments of orocaecal transit time and stool weight.

Department of Gastroenterology,

L J D O’DONNELL

Mayo General Hospital,

Castlebar,

Ireland

Email:lodonnell@anu.ie

1 Steiner CA, Bass EB, Talamini MA, et al. Surgical rates and operative mortality for open and laparoscpic cholecystectomy in Maryland. N Engl f Med 1994;330:403-8

2 Al-Mulhim AA, Al-Ali AA, Albar AA, et al. Increased rate of cholecystectomy after introduction of laparoscopic cholecystectomy in Saudi Arabia. World 7 Surg 1999;23:458-62.

3 Fendrick AM, Gleeson SP, Cabana MD, et al. Asymptomatic gallstones revisited. Is there a role for laparoscopic cholecystectomy? Arch Family Med revisited. Is there

4 Qureshi MA, Brindley NM, Osborne DH, et al. Post cholecystectomy symptoms after laparoscopic cholecystectomy. Ann Royal College Surg Engl symptoms after lapary

5 Vander Velpen GC, Shimi SM, Cuschieri A. Outcome after cholecystectomy for symptomatic gallstone disease and effect of surgical access: laparoscopic v open. Gut 1993;34:1448-51

6 Luman W, Adams WH, Nixon SN, et al. Incidence of persistent symptoms after laparoscopic cholecystectomy: a prospective study. Gut 1996;39:863-6.

7 Jorgensen T. Abdominal symptoms and gallstone disease: an epidemiological investigation. Hepatology 1989;9:856-60.

8 Dunn D, Nair R, Fowler S, et al. Laparoscopic cholecystectomy in England and Wales: results of an audit by the Royal College of Surgeons of England. Ann Royal College Surg Engl 1994;76:269-75.

9 Fort JM, Azpiroz F, Casellas F, et al. Bowel habit after cholecystectomy: physiological changes and clinical implications. Gastroenterology 1996;111: physiolog 